\title{
Recommendations on screening for cognitive impairment in older adults
}

\author{
Canadian Task Force on Preventive Health Care* \\ $C M A J$ podcasts: author interview at https://soundcloud.com/cmajpodcasts/141165-guide
}

See also www.cmaj.ca/lookup/doi/10.1503/cmaj.151411 and www.cmajopen.ca/content/3/4/E419

A s people age, changes to the structure and the function of the brain may result in cognitive decline. These changes, however, do not equally affect all cognitive domains or all people. Older adults may perform as well as younger adults in some or all cognitive domains, and some may even perform better. ${ }^{1}$ The most common cognitive functions affected by age are memory and perception, which in some cases may have an impact on more complex cognitive functions such as decision-making and language. ${ }^{1}$

Cognitive impairment occurs in a continuum, starting with aging-related cognitive decline, transitioning to mild cognitive impairment and ending with dementia. Mild cognitive impairment is noticeable but does not substantially affect daily function, whereas dementia involves cognitive changes that are severe enough to affect daily function. ${ }^{2,3}$ Although some people with mild cognitive impairment may be at higher risk of dementia than others with the diagnosis, over time some will remain stable and a few will show improvements in their cognitive abilities. ${ }^{1}$ The incidence of dementia among Canadian adults aged 65-79 years is 43 per 1000 population and rises with age (to 212 per 1000 among those aged 85 or older). ${ }^{4}$ The reported prevalence of mild cognitive impairment varies because of several factors, such as the diagnostic test score (i.e., cut-off) used to define mild cognitive impairment, age at the initial assessment and length of follow-up. ${ }^{5}$ Published Canadian cohort prevalence rates for mild cognitive impairment are not available. Studies from the United States have reported prevalence ranging from $9.9 \%$ to $35.2 \%$ among adults aged 70 or older. ${ }^{6-8}$

Cognitive impairment is commonly assessed using screening tools such as the Mini-Mental State Examination (MMSE), the Montreal Cognitive Assessment (MoCA) and the cognitive subscale of the Alzheimer's Disease Assessment Scale (ADAS-cog). ${ }^{9-11}$ The MMSE has a raw score range of 0 to 30 and typically uses a cut-off of scores below 23 to suggest likely cognitive impairment, ${ }^{12}$ although the specific cut-off that is recommended varies based on age and education level. ${ }^{11}$ Clinical significance is considered to be a change in score of 1.4 to 3 points. A systematic review ${ }^{11}$ reported that a large body of studies suggested a general cut-off between 23 and 24 or between 24 and 25 to screen for dementia, which could be appropriate for most primary care populations. The review also reported a cut-off of 27 or 28 points for MMSE to detect mild cognitive impairment (with a low and wide range of sensitivity rates). ${ }^{11}$ However, these reported cut-offs were based on a limited subset of studies that used different definitions and had different prevalence rates of underlying mild cognitive impairment.

The MoCA is also scored out of 30 and provides interpretive guidance as follows: mild cognitive impairment (scores of 18-26), moderate
Competing interests: None of the authors (members of the guideline writing group) have declared competing interests.

This article has been peer reviewed.

*The authors of this article are listed at the end of the article, under "guideline writing group."

The complete list of current members of the Canadian Task Force on Preventive Health Care is available at http://canadiantaskforce.ca/ about-us/members/

Correspondence to:

Canadian Task Force on Preventive Health Care, info@canadiantaskforce.ca

CMAJ 2016. DOI:10.1503 /cmaj.141165

\section{Ker pOINTS}

- No randomized trials have evaluated the benefits of screening for cognitive impairment.

- Available data suggest that pharmacologic treatments are not effective in people with mild cognitive impairment and that nonpharmacologic therapies (e.g., exercise, cognitive training and rehabilitation) produce only small benefits, which do not appear to be clinically significant.

- Existing studies suggest that about 1 in 10 people without cognitive impairment may erroneously screen positive for mild cognitive impairment using the Mini-Mental State Examination and that 1 in 4 people may incorrectly be classified as positive using the Montreal Cognitive Assessment tool.

- The task force recommendation against screening asymptomatic adults 65 years of age and older for cognitive impairment is based on the lack of high-quality studies evaluating the benefits and harms of screening for cognitive impairment, on evidence showing that treatment of mild cognitive impairment does not produce clinically meaningful benefit and on the potentially high rate of false-positive screens that may occur.

- The strong recommendation against screening asymptomatic adults 65 years of age and older for cognitive impairment implies that the task force is confident that most individuals will be best served by the recommended course of action.

- Practitioners should consider cognitive assessment for patients with signs and symptoms of impairment or when family members or patients express concerns about potential cognitive decline. 
cognitive impairment (scores of 10-17) and severe impairment (scores less than 10). ${ }^{13}$

The ADAS-cog is a cognitive testing instrument not normally used in practice but often used in research studies. It consists of 11 tasks measuring disturbances of memory, language, praxis (i.e., application or use of specific knowledge or skills such as drawing geometric figures or fitting a page into an envelope), attention and other cognitive abilities. ${ }^{9}$ A change in the ADAS-cog score of 4 points is considered by many clinical experts to represent a clinically important change. The MMSE and the MoCA tools are commonly used by Canadian clinicians in clinical practice. ${ }^{14}$

Treatments include medications such as cholinesterase inhibitors (i.e., donepezil, rivastigmine and galantamine), dietary supplements and vitamins, and nonpharmacologic interventions such as exercise, and cognitive training and rehabilitation. ${ }^{10}$ Provincial payment for the medications used in primary care practice is often linked to cognitive assessment scores measured by the screening instruments.

The objective of this guideline, which updates the 2001 Canadian Task Force on Preventive Health Care recommendations, ${ }^{15}$ is to provide evidence-based recommendations on screening for cognitive impairment in adults. The guideline focuses on screening asymptomatic adults. This recommendation does not apply to men and women who are concerned about their own cognitive performance (i.e., patients who report cognitive changes to their clinician or others) or who are suspected of having mild cognitive impairment or dementia by clinicians or nonclinicians (i.e., caregivers, family or friends) and who have symptoms suggestive of mild cognitive impairment or dementia (e.g., loss of memory, language, attention, visuospatial or executive functioning, or behavioural or psychological symptoms that may mildly or substantially affect a patient's day-to-day life or usual activities).

\section{Methods}

The Canadian Task Force on Preventive Health Care is an independent panel of volunteer clinicians and methodologists that makes recommendations about clinical manoeuvres aimed at primary and secondary prevention (www.canadian taskforce.ca). The development of these recommendations was led by a workgroup of eight members of the task force and scientific staff at the Public Health Agency of Canada. The task force established this topic as a priority based on the potential to decrease inconsistencies in screening in primary care practice and a need to determine whether benefits of screening outweigh harms.

The US Preventive Services Task Force recently published a systematic review on screening and treatment for cognitive impairment. ${ }^{11}$ Initially, the Canadian task force updated the US task force review, assessing the effects of screening for $\operatorname{cog}$ nitive impairment on health outcomes. ${ }^{9}$ The Evidence Review and Synthesis Centre at McMaster University independently conducted the systematic review evaluating the effectiveness of screening for cognitive impairment (Appendix 1, available at www.cmaj.ca/lookup/suppl/doi:10.1503/ cmaj.141165/-/DC1). The systematic review included the following patient-important outcomes from the US task force review: safety, healthrelated quality of life (HRQoL), cognitive function or decline, unanticipated health care utilization, independent living, medication adherence or errors, and other symptoms (e.g., insomnia, depression or agitation). Caregiver outcomes included HRQoL and caregiver burden. The study selection included randomized controlled trials (RCTs) published from Dec. 8, 2012, to Nov. 7, 2014, in the databases MEDLINE, PsychINFO and the Cochrane Central Register of Controlled Trials. No trials were identified that investigated the benefits of screening for cognitive impairment. The results are shown in Appendix 1.

Given that the Evidence Review and Synthesis Centre found no evidence on the benefits or harms of screening, the task force decided to also review the literature on the effectiveness of treatment interventions for mild cognitive impairment to inform the screening guidelines. The workgroup established the research questions and the analytical framework for the guideline (Appendix 2, available at www.cmaj.ca/lookup/suppl/doi:10.1503/cmaj $.141165 /$-/DC1), which were incorporated into the review protocol (registered with PROSPERO, at www.crd.york.ac.uk/PROSPERO; registration no. CRD42014015431)..$^{10}$ This second systematic review updated the search conducted for the US task force systematic review on the effectiveness of treatment; ${ }^{11}$ however, the US task force's inclusion criteria were modified by the Canadian Task Force on Preventive Health Care to examine the effectiveness of treatment only in individuals with a diagnosis of mild cognitive impairment. Our assumptions were that, if clinicians are able to identify individuals with mild cognitive impairment early through screening and either slow down or stop its progression through effective treatment, the incidence of cognitive impairment (measured through cognition, function, behaviour and global status) may decline. Also, if individuals are identified at the stage of mild cognitive impairment, when their comprehension 
and decision-making capacity and autonomy are not affected, they will have the opportunity to plan for the future in different areas of their lives (e.g., medical, legal, financial), which may ultimately improve other patient-important outcomes, such as a reduction of caregiver burden.

The Evidence Review and Synthesis Centre review on the effectiveness of treatment interventions for mild cognitive impairment was conducted in accordance with the final, peer-reviewed protocol. The search included RCTs published from December 2012 to December 2014 that were conducted in primary care settings. A clinical expert was consulted throughout the process. The task force workgroup selected outcomes important to patients and the scales used to measure such outcomes from those selected and prioritized by Canadian clinicians and policy-makers. ${ }^{14}$ These outcomes included cognition (measured with the MMSE or ADAS-cog); function (measured by Alzheimer's disease Cooperative Study activities of daily living inventory [ADL]); behaviour (measured by Neuropsychiatric Inventory [NPI]); global status (measured by Clinician's Interview-based Impression of Change plus Caregiver [CGIC$\mathrm{MCI}]$ ); and mortality. ${ }^{10}$ The harms for treatment were identified by the guideline working group, which included serious adverse events (i.e., hospital admission or death) and psychosocial harms (e.g., lack of independence, stress, depression). ${ }^{10}$

Studies that did not use the measures of cognition prioritized by the Canadian clinicians and policy-makers ${ }^{14}$ were excluded from the systematic review. Duration of intervention had to be at least six months. ${ }^{10}$ As in the review by Tricco and colleagues, ${ }^{14}$ the results were presented separately for each outcome measure. Treatments included all pharmacologic interventions approved for use in Canada (e.g., cholinesterase inhibitors, such as donepezil, rivastigmine and galantamine), dietary supplements or vitamins and nonpharmacologic interventions (e.g., exercise, cognitive training and rehabilitation).

The task force workgroup decided to treat the key question regarding the accuracy of screening tools (key question 6 in Appendix 2) as a contextual question. This was because there were no trials of screening programs and there was evidence that treatment of mild cognitive impairment does not produce clinically meaningful benefit. Thus, the accuracy of potential screening tools was less important for determining an overall recommendation, but it was still important to understand the likely burden of false-positive results if screening were to be implemented. As such, a systematic review of the evidence on diagnostic test properties was not conducted. Instead, two recent high-quality systematic reviews $^{11,16}$ (AMSTAR [A Measurement Tool to Assess Systematic Reviews] scores of 9 and 10, respectively) were used to report on the sensitivity and specificity of screening tools. As well, falsepositive rates were reported, which was defined as the proportion of people without cognitive impairment who would be incorrectly classified as possible cases (calculated as 1 - specificity).

More information about the task force's methods can be found elsewhere, ${ }^{17}$ on the task force website (http://canadiantaskforce.ca/methods/ methods-manual) and in Box $1 .^{18}$

\section{Box 1: Grading of recommendations}

- Recommendations are graded according to the Grading of Recommendations Assessment, Development and Evaluation (GRADE) system. ${ }^{18}$ GRADE offers two strengths of recommendation: strong and weak. The strength of recommendations is based on the quality of supporting evidence, the degree of uncertainty about the balance between desirable and undesirable effects, the degree of uncertainty or variability in values and preferences, and the degree of uncertainty about whether the intervention represents a wise use of resources.

- Strong recommendations are those for which the Canadian Task Force on Preventive Health Care is confident that the desirable effects of an intervention outweigh its undesirable effects (strong recommendation for an intervention) or that the undesirable effects of an intervention outweigh its desirable effects (strong recommendation against an intervention). A strong recommendation implies that most individuals will be best served by the recommended course of action.

- Weak recommendations are those for which the desirable effects probably outweigh the undesirable effects (weak recommendation for an intervention) or the undesirable effects probably outweigh the desirable effects (weak recommendation against an intervention) but appreciable uncertainty exists. A weak recommendation implies that most people would want the recommended course of action but that many would not. For clinicians, this means they must recognize that different choices will be appropriate for each individual, and they must help each person arrive at a management decision consistent with his or her values and preferences. Policy-making will require substantial debate and involvement of various stakeholders. Weak recommendations result when the balance between desirable and undesirable effects is small, the quality of evidence is lower, or there is more variability in the values and preferences of patients.

- The quality of evidence is graded as high, moderate, low or very low, based on how likely further research is to change our confidence in the estimate of effect. 


\section{Recommendation}

We recommend not screening asymptomatic older adults ( $\geq 65$ yr) for cognitive impairment. (Strong recommendation, low-quality evidence.)

A summary of the recommendation is shown in Box 2. No evidence was found on the effectiveness of screening, as discussed in Appendix 1. The summary of evidence for benefits of treatment is shown in Table $1 .{ }^{19-30}$ A summary of the GRADE decision table can be found in Appendix 3 (available at www.cmaj.ca/lookup/suppl/doi:10.1503/ cmaj.141165/-/DC1), with detailed tables provided in the accompanying evidence review. ${ }^{10}$

The recommendation applies to communitydwelling adults 65 years of age or older who do not have noticeable symptoms suggestive of mild cognitive impairment or dementia. This recommendation does not apply to men or women who are concerned about their own cognitive performance (i.e., patients who have raised complaints about cognitive changes with their clinician or others) or who are suspected of having mild cognitive impairment or dementia by clinicians or nonclinicians (caregivers, family or friends) and/or have symptoms suggestive of mild cognitive impairment or dementia (e.g., loss of memory, language, attention, visuospatial or executive functioning, or behavioural or psychological symptoms that may either mildly or substantially affect a patient's dayto-day life or usual activities).

\section{Benefits of screening and treatment}

The evidence review identified no trials that examined the effectiveness of screening for $\operatorname{cog}$ nitive impairment on patient outcomes (function, quality of life, health care utilization and safety), family and caregiver outcomes (quality of life, caregiver burden) or societal outcomes (safety) $)^{10}$ (Appendix 1). The review identified 12 RCTs

\section{Box 2: Summary of recommendations for} clinicians and policy-makers

We recommend not screening asymptomatic adults 65 years of age or older for cognitive impairment. (Strong recommendation, lowquality evidence.)

The recommendation applies to communitydwelling adults 65 years of age or older in whom cognitive impairment has not been identified as a specific concern. This recommendation does not apply to men and women who have symptoms suggestive of cognitive impairment (e.g., loss of memory, language, attention, visuospatial or executive functioning, or behavioural or psychological symptoms) or who are suspected of having cognitive impairment by clinicians, family or friends. that examined the effects of treatment interventions for mild cognitive impairment on cognition, function, behaviour and global status. No studies were identified that examined the effect of treatment interventions on mortality.

For all results on the outcome of cognition reported below, it is important to note that negative and positive effects are outcome-measure dependent. For MMSE, increases in score (positive values) indicate an improvement; however, for ADAS-cog, decreases in score (negative values) indicate an improvement.

\section{Cholinesterase inhibitors}

The systematic review concluded that cholinesterase inhibitors did not improve cognition in patients with mild cognitive impairment when measured with ADAS-cog or with MMSE (Appendix 4, available at www.cmaj.ca/lookup/ suppl/doi:10.1503/cmaj.141165/-/DC1). ${ }^{10}$ More specifically, a meta-analysis of four trials ${ }^{19-22}$ ( $n=4188)$ evaluating the benefits of cholinesterase inhibitors found no statistically significant effects of these treatments on cognition measured with ADAS-cog (mean difference $-0.33,95 \%$ confidence interval $[\mathrm{CI}]-0.73$ to 0.06 ). Similarly, no improvement was observed when measuring cognition with MMSE ( 3 trials, $n=2287$; mean difference $0.17,95 \% \mathrm{CI}-0.13$ to 0.47 ). ${ }^{19-21}$

The systematic review also showed that cholinesterase inhibitors did not improve behaviour, global status or function when measured with NPI, CGIC-MCI and ADL respectively. ${ }^{10}$ Two trials $^{19,21}(n=1775)$ showed no significant effects on behaviour (measured with NPI: mean difference $0.12,95 \%$ CI -0.93 to 1.17 ), and one trial ${ }^{19}$ $(n=757)$ showed no significant effect on global status (measured with CGIC-MCI: mean difference $0.00,95 \% \mathrm{CI}-0.28$ to 0.28$)$. Three trials $\mathrm{s}^{20-22}$ $(n=3431)$ showed no significant effect on function (measured with ADL: mean difference 0.20, $95 \% \mathrm{CI}-0.28$ to 0.69 ). No studies examining the effect of treatment with memantine (with at least six months of post-baseline data) were identified in the systematic review.

\section{Dietary supplements and vitamins}

The systematic review concluded that dietary supplements and vitamins did not improve cognition (Appendix 4)..$^{10}$ Four RCTs were identified evaluating the effect of dietary supplements or vitamins. ${ }^{20,23-25}$ Of these trials, two ${ }^{20,25}$ examined the effects of vitamin $\mathrm{E}$ (either with vitamin $\mathrm{C}$ or with a multivitamin) on cognition or function, one $^{24}$ examined the effects of fish oils, and another ${ }^{23}$ examined the effect of vitamin B on cognition. No data were identified that examined the use of dietary supplements or vitamins on 
behaviour, global status or mortality. None of the four studies showed an improvement in cognition when treating mild cognitive impairment with dietary supplements or vitamins measured with ADAS-Cog (one trial, ${ }^{20} n=516$; mean difference $0.85,95 \% \mathrm{CI}-0.32$ to 2.02 [negative scores indicate improvement of symptoms]) or measured with MMSE (four trials, ${ }^{20,23-25} n=1030$; mean difference $0.20,95 \% \mathrm{CI}-0.04$ to 0.43 [positive scores indicate improvement of symptoms]). ${ }^{10}$

The systematic review also showed that dietary supplements and vitamins did not improve function measured with ADL (one trial, ${ }^{20} n=516$; mean difference $0.76,95 \% \mathrm{CI}$ -0.78 to 2.29 . $)^{10}$

\section{Nonpharmacologic interventions}

The systematic review identified five studies ${ }^{26-30}$ $(n=408)$ that examined the effects on cognition of nonpharmacologic interventions (Appendix 4), including exercise, a holistic cognitive rehabilitation program and a multimodal intervention with stimulation and cognitive training sessions. ${ }^{10}$ Using ADAS-Cog to measure cognition, one $\operatorname{trial}^{28}(n=92)$ showed no significant differences between the intervention and control groups (mean difference $-0.60,95 \%$ CI -1.44 to 0.24 [negative scores indicate improvement of symptoms]). However, a meta-analysis of the five trials $(n=408)$ that used MMSE to measure $\operatorname{cognition}^{26-30}$ found a statistically significant effect of the behavioural interventions (mean difference $1.01,95 \%$ CI 0.25 to 1.77 ). Clinical significance on the MMSE is often defined as a change of 1.4 to 3 points; therefore, this difference is not considered clinically significant. ${ }^{31}$

\section{Harms}

No studies were identified by the Evidence Review and Synthesis Centre on the harms of screening (shown in Appendix 1).

The systematic review for treatment also used RCTs to examine harms associated with the treatment of mild cognitive impairment and found no evidence that pharmacologic treatments were associated with an increased number of serious adverse events or psychosocial harms (e.g., depression and lack of independence) compared with controls. ${ }^{10}$ Seven RCTs ${ }^{20,28,30,32-35}$ that examined the effects of dietary supplements or vitamins or the effects of nonpharmacologic treatments reported that no serious adverse events occurred.

\section{Rationale for recommendation}

The findings of the evidence review highlight the lack of high-quality studies evaluating the benefits and harms of screening for cognitive impairment and the lack of effective treatments for mild cognitive impairment. If screening for cognitive impairment were to be conducted in the asymptomatic general population, most cases detected would likely be mild cognitive impairment, not dementia. Therefore, the task force felt it was important to examine the effectiveness of treatments for mild cognitive impairment.

Given the lack of evidence on the efficacy of screening for cognitive impairment and the lack of clinically effective treatments for mild cognitive impairment, the task force felt a strong recommendation against screening asymptomatic patients for cognitive impairment was warranted. A strong recommendation means that the task force is confident that the benefits do not outweigh the risks of screening most asymptomatic individuals 65 years of age and older.

This recommendation places a relatively higher value on the lack of evidence evaluating the benefits and harms of screening, evidence showing that pharmacologic treatment is not effective, and evidence showing a small benefit of nonpharmacologic treatment, which was not clinically significant. This recommendation places a relatively lower value on limited evidence about patient preferences, which showed that about $50 \%$ of first-degree relatives of people with diagnosed cognitive impairment may be willing to be screened (see "Patient values and preferences"), and limited evidence showing that treatment of mild cognitive impairment is not associated with an increase of serious adverse events or psychosocial harms.

The evidence supporting this recommendation is rated overall as low quality because no evidence was found on the effectiveness of screening, because the RCTs evaluating the effectiveness of pharmacologic treatments were all rated as low quality, and because the five RCTs showing a small benefit (not clinically significant) of nonpharmacologic interventions on cognition, although rated as moderate quality, were downgraded because of serious study limitations.

\section{Considerations for implementation}

The task force has developed tools to help practitioners interpret these recommendations for their patients (available at http://canadiantaskforce.ca/ ctfphc-guidelines/2015-cognitive-impairment-2). The task force uses a rigorous and collaborative usability testing process to develop knowledge translation tools targeting various end-user groups (e.g., clinicians and patients) to accompany its guidelines. Tools are informed by feedback from clinicians and patients obtained in interviews or focus groups. Although we recommend against screening for cognitive impairment, practitioners 
Table 1 (part 1 of 2): GRADE table of beneficial effects of treatment interventions for mild cognitive impairment on cognition ${ }^{19-29,30}$



Note: ADAS-cog = cognitive subscale of the Alzheimer's Disease Assessment Scale, $\mathrm{Cl}=$ confidence interval, MMSE = Mini-Mental State Examination, RT = randomized trial. ${ }^{*}$ Clinical significance is considered to be a change in score of between 1.4 to 3 points on MMSE and 4 points on ADAS-Cog. Negative and positive effects are outcome-measure dependent. For MMSE, increases in score (positive values) indicate an improvement of symptoms; however, for ADAS-cog, decreases in score (negative values) indicate an improvement.

tGrading of Recommendations, Assessment, Development and Evaluation (GRADE) rates the continuum of quality of evidence in 4 categories of high, moderate, low or very low. All data can be found in the accompanying evidence review. ${ }^{10}$

asing Cochrane's risk-of-bias tool, for this outcome 1 study was rated as low and 2 studies were rated as unclear risk. Across the studies, there was a lack of certainty (unclear ratings) regarding sequence generation (33\%) and allocation concealment (33\%), and a high risk of bias associated with incomplete outcome reporting (33\%) and other sources of bias $(67 \%$; i.e., industry funding, baseline differences between groups, insufficiently powered and/or sample size $<30$ per arm). Given that most of the information is from studies at moderate risk of bias, this body of evidence was downgraded for serious study limitations.

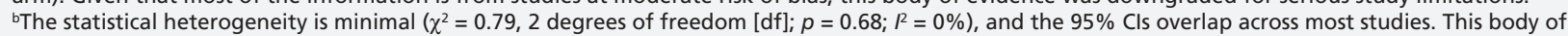
evidence was not downgraded for inconsistency.

Three randomized controlled trials (RCTs) provided data for this outcome. All studies included mixed sex samples. The mean age across studies ranged from 69 to 74 years. The intervention arm received donepezil ( $10 \mathrm{mg} / \mathrm{d})$ in 2 studies and rivastigmine (3-12 $\mathrm{mg} / \mathrm{d})$ in 1 study; all control groups received placebo. One study was conducted in the United States and Canada, 1 in the US and 1 study in 14 countries. All studies were published from 2005 to 2009 . The length of intervention across studies ranged from 11 to 48 months. There were no serious concerns regarding indirectness for this body of evidence, and it was not downgraded.

${ }^{\mathrm{d}}$ The sample size is adequate (i.e., > 300 [1140 intervention arm, 1147 control arm]), but the pooled effect estimate is not precise and the $95 \% \mathrm{Cl}$ includes the null value " 0 " (mean difference $=0.17$ [95\% $\mathrm{Cl}-0.13$ to 0.47$]$ ). This body of evidence was downgraded for serious concerns regarding imprecision.

eThere were too few studies $(<10)$ to assess publication bias.

fUsing Cochrane's risk-of-bias tool, for this outcome 1 study was rated as low and 3 studies were rated as unclear risk. Across studies, there was a lack of certainty (unclear ratings) regarding sequence generation (50\%) and allocation concealment (50\%), and a high risk of bias associated with incomplete outcome reporting $(25 \%)$ and other sources of bias ( $75 \%$; i.e., industry funding, baseline differences between groups, insufficiently powered and/or sample size < 30 per arm). Given that most of the information is from studies at moderate risk of bias, this body of evidence was downgraded for serious study limitations.

${ }^{9}$ The statistical heterogeneity is minimal $\left(\chi^{2}=4.63,4 \mathrm{df} ; p=0.33 ; l^{2}=14 \%\right)$, and the $95 \%$ Cls overlap across most studies. This body of evidence was not downgraded for inconsistency.

hFour RCTs provided data for this outcome. All studies included mixed sex samples. The mean age across studies ranged from 69 to 74 years. The intervention arm received donepezil $(10 \mathrm{mg} / \mathrm{d})$ in 2 studies, rivastigmine $(3-12 \mathrm{mg} / \mathrm{d})$ in 1 study and galantamine $(16-24 \mathrm{mg} / \mathrm{d})$ in $1 \mathrm{study}$. The control group across all studies received placebo. Two studies were conducted in the US and Canada, 1 study in the US and 1 study in 14 countries. All studies were published from 2005 to 2009 . The length of intervention across studies ranged from 11 to 48 months. There were no serious concerns regarding indirectness for this body of evidence, and it was not downgraded. 


\section{Table 1 (part 2 of 2): GRADE table of beneficial effects of treatment interventions for mild cognitive impairment on cognition ${ }^{19-29,30}$}

'The sample size is adequate (i.e., > 300 [2078 intervention arm, 2110 control arm]), but the pooled effect estimate is not precise and the $95 \%$ Cl includes the null value " 0 " (mean difference $=-0.33[95 \% \mathrm{Cl}-0.73$ to 0.06$]$ ). This body of evidence was downgraded for serious concerns regarding imprecision.

iUsing Cochrane's risk-of-bias tool, for this outcome 1 study was rated as low and 1 as unclear risk. Across studies, there was a lack of certainty (unclear ratings) regarding sequence generation $(50 \%)$ and allocation concealment $(50 \%)$, and a high risk associated with other sources of bias $(50 \%$; i.e., industry funding, baseline differences between groups, insufficiently powered and/or sample size $<30$ per arm). Given that most of the information is from studies at moderate risk of bias, this body of evidence was downgraded for serious study limitations.

${ }^{k}$ The statistical heterogeneity is minimal $\left(\chi^{2}=0.58,1 \mathrm{df} ; p=0.44 ; R=0 \%\right)$, and the $95 \%$ Cls overlap across studies. This body of evidence was not downgraded for inconsistency.

'Two RCTs provided data for this outcome. Both studies included mixed sex samples. The mean age across studies ranged from 70 to 74 years. The intervention arm received donepezil ( $10 \mathrm{mg} / \mathrm{d})$ and the control group received placebo. One study was conducted in the US, and 1 in the US and Canada. One study was published in 2005 and 1 in 2009. The length of intervention across studies ranged from 11 to 36 months. There were no serious concerns regarding indirectness for this body of evidence, and it was not downgraded.

mThe sample size is adequate (i.e., > 300 [632 intervention arm, 637 control arm]), but the pooled effect estimate is not precise and the $95 \%$ Cl includes the null value " 0 " (mean difference $=0.24$ [95\% Cl -0.19 to 0.67$]$ ). This body of evidence was downgraded for serious concerns regarding imprecision.

${ }^{n}$ The statistical heterogeneity is minimal $\left(\chi^{2}=1.48,1 \mathrm{df} ; p=0.22 ; R^{2}=33 \%\right)$, and the $95 \%$ Cls overlap across studies. This body of evidence was not downgraded for inconsistency.

'The sample size is adequate (i.e., > 300 [632 intervention arm, 637 control arm]), but the pooled effect estimate is not precise and the $95 \% \mathrm{Cl}$ includes the null value " 0 " (mean difference -0.60 [95\% Cl -1.35 to 0.15$]$ ). This body of evidence was downgraded for serious concerns regarding imprecision.

pUsing Cochrane's risk-of-bias tool, for this outcome the included study was rated as unclear risk. There was a high risk of bias associated with incomplete outcome reporting and other sources of bias (i.e., industry funding, baseline differences between groups, insufficiently powered and/or sample size < 30 per arm). Given that the information is from a study with moderate risk of bias, this body of evidence was downgraded for serious study limitations.

"The statistical heterogeneity across studies could not be assessed owing to only 1 study providing data for this outcome.

rOne RCT provided data for this outcome. The study included a mixed sex sample. The mean age was 70.6 years for the intervention group and 70.3 years for the control group. The intervention arm received rivastigmine (3-12 mg/d); the control group received placebo. The study was conducted in 14 countries and published in 2007. The length of intervention was 48 months. There were no serious concerns regarding indirectness for this body of evidence, and it was not downgraded. 'The sample size is adequate (i.e., > 300 [508 intervention arm, 510 control arm]), but the pooled effect estimate is not precise and the $95 \% \mathrm{Cl}$ includes the null value " 0 " (mean difference $=0.10[95 \% \mathrm{Cl}-0.32$ to 0.52$]$ ). This body of evidence was downgraded for serious concerns regarding imprecision.

tThe sample size is adequate (i.e., > 300 [508 intervention arm, 510 control arm]), but the pooled effect estimate is not precise and the $95 \% \mathrm{Cl}$ includes the null value " 0 " (mean difference $=0.0[95 \% \mathrm{Cl}-0.80$ to 0.80$]$ ). This body of evidence was downgraded for serious concerns regarding imprecision.

using Cochrane's risk-of-bias tool, for this outcome the included study was rated as unclear risk. There was a lack of certainty (unclear ratings) regarding sequence generation and allocation concealment, and a high risk of bias associated with incomplete outcome reporting and other sources of bias (i.e., industry funding, baseline differences between groups, insufficiently powered and/or sample size $<30$ per arm). Given that most of the information is from studies at moderate risk of bias, this body of evidence was downgraded for serious study limitations.

vOne RCT provided data for this outcome. The study included a mixed sex sample. The study included results from 2 trials: in the first trial, the mean age was 69.2 years in the intervention group and 70.1 years in the control group; the mean age was 70.6 years and 70.9 years, respectively, in the second trial. The intervention arm received galantamine (16-24 mg/d) in both trials; the control group received placebo. The study was conducted in the US and Canada and published in 2008 . The length of intervention was 24 months. There were no serious concerns regarding indirectness for this body of evidence and was not downgraded.

wThe sample size is adequate (i.e., > 300 [938 intervention arm, 963 control arm]), but the pooled effect estimate is not precise and the $95 \%$ Cl includes the null value " 0 " (mean difference $=-0.21[95 \% \mathrm{Cl}-0.80$ to 0.38$]$ ). This body of evidence was downgraded for serious concerns regarding imprecision.

'Using Cochrane's risk-of-bias tool, for this outcome 1 study was rated as low and 3 studies were rated as unclear risk. Across studies, there was a lack of certainty (unclear ratings) regarding sequence generation $(50 \%)$ and allocation concealment $(75 \%)$, and a high risk associated with other sources of bias ( $25 \%$; i.e., industry funding, baseline differences between groups, insufficiently powered and/or sample size $<30$ per arm). Given that most of the information is from studies at moderate risk of bias, this body of evidence was downgraded for serious study limitations.

yThe statistical heterogeneity is minimal $\left(\chi^{2}=1.36,3 \mathrm{df} ; p=0.71 ; R^{2}=0 \%\right)$, and the $95 \%$ Cls overlap across most studies. This body of evidence was not downgraded for inconsistency.

'Four RCTs provided data for this outcome. All studies included mixed sex samples. The mean age across studies ranged from 66 to 77 years. The intervention arm received vitamin $\mathrm{E}$ in 1 study, vitamin $\mathrm{E}$ and folic acid in 1 study, docosahexaenoic acid (DHA; fish oil) in 1 study, and vitamins $\mathrm{E}$ and $\mathrm{C}$ in 1 study; all control groups received placebo. One study was conducted in the US and Canada, 1 in the United Kingdom, 1 in Malaysia and 1 in Iran. All studies were published from 2005 to 2014. The length of intervention across studies ranged from 12 to 36 months. There were no serious concerns regarding indirectness for this body of evidence, and it was not downgraded.

AThe sample size is adequate (i.e., > 300 [511 intervention arm, 519 control arm]), but the pooled effect estimate is not precise and the $95 \%$ Cls includes the null value " 0 " (mean difference $=0.20$ [95\% Cl -0.04 to 0.43$]$ ). This body of evidence was downgraded for serious concerns regarding imprecision.

BUsing Cochrane's risk-of-bias tool, for this outcome the included study was rated as unclear risk. There was a lack of certainty (unclear ratings) regarding sequence generation and allocation concealment. Given that the information is from a study with moderate risk of bias, this body of evidence was downgraded for serious study limitations.

'One RCT provided data for this outcome. The study included a mixed sex sample. The mean age was 72.8 years for the intervention group and 72.9 years for the control group. The intervention arm received donepezil $(10 \mathrm{mg} / \mathrm{d})$; the control group received placebo. The study was conducted in the US and Canada and was published in 2005. The length of intervention was 36 months. There were no serious concerns regarding indirectness for this body of evidence, and it was not downgraded. DThe sample size is not adequate (i.e., < 300 [257 intervention arm, 259 control arm]), and the pooled effect estimate is not precise with a $95 \%$ Cl that includes the null value " 0 " (mean difference $=0.85$ [ $95 \% \mathrm{Cl}-0.32$ to 2.02]). This body of evidence was downgraded for serious concerns regarding imprecision.

EUsing Cochrane's risk-of-bias tool, for this outcome 1 study was rated as low and 4 studies were rated as unclear risk. Across studies, there was a lack of certainty (unclear ratings) regarding sequence generation ( $80 \%)$, allocation concealment $(80 \%)$, blinding (20\%), incomplete outcome reporting (20\%) and other sources of bias $(20 \%)$, and a high risk of bias associated with blinding ( $20 \%)$, incomplete outcome reporting ( $20 \%)$ and other sources of bias ( $40 \%$; i.e., industry funding, baseline differences between groups, insufficiently powered and/or sample size $<30$ per arm). Given that most of the information is from studies at moderate risk of bias, this body of evidence was downgraded for serious study limitations.

FThe statistical heterogeneity is high $\left(\chi^{2}=16.92,4 \mathrm{df} ; p=0.002 ; \ell^{2}=76 \%\right)$, but the direction of the effect is consistent across studies and the $95 \%$ Cls overlap across most studies. The statistical heterogeneity is most likely due to small versus large treatment effects observed across studies. This body of evidence was not downgraded for inconsistency.

GFive RCTs provided data for this outcome. All studies included mixed sex samples. The mean age across studies ranged from 65 to 77 years. The intervention arm received multicomponent exercise programs in 3 studies and cognitive training and rehabilitation in 2 studies; the control group across studies received no therapy, wait list, or minimal contact involving education about health promotion. Two studies were conducted in Japan, 1 in China, 1 in Greece and 1 in Argentina. All studies were published from 2009 to 2014. The length of intervention across 4 studies ranged from 6 to 12 months. There were no serious concerns regarding indirectness for this body of evidence, and it was not downgraded.

${ }^{H}$ The sample size is not adequate (i.e., < 300 [221 intervention arm, 187 control arm]), but the pooled effect estimate is precise with a narrow $95 \%$ Cl (mean difference $=1.01$ [95\% Cl 0.25 to 1.77$]$ ). This body of evidence was not downgraded for serious concerns regarding imprecision.

'Using Cochrane's risk-of-bias tool, for this outcome the included study was rated as low risk. There were no serious concerns regarding risk of bias, and this body of evidence was not downgraded for serious study limitations.

'One RCT provided data for this outcome. The study included a mixed sex sample. The mean age was 74.8 years for the intervention group and 75.8 years for the control group. The intervention arm received a multicomponent exercise program biweekly; the control group received minimal contact with 2 education classes about health promotion. The study was conducted in Japan and published in 2013. The length of intervention was 6 months. There were no serious concerns regarding indirectness for this body of evidence, and it was not downgraded.

KThe sample size is not adequate (i.e., < 300 [47 intervention arm, 45 control arm]), and the pooled effect estimate is not precise with a $95 \%$ Cl that includes the null value " 0 " (mean difference $=-0.60[95 \% \mathrm{Cl}-1.44$ to 0.24$]$ ). This body of evidence was downgraded for serious concerns regarding imprecision 
should examine and assess cognitive functions and functional autonomy whenever a patient presents with signs and symptoms of impairment or when family members or patients express concerns about potential cognitive decline. ${ }^{11,36}$

It is difficult to establish the potential value of screening in older populations, such as people over 85 years of age. The prevalence of mild cognitive impairment and dementia increases in older groups (e.g., > 85). ${ }^{4}$ However, given the lack of high-quality evidence showing the effectiveness of treatment and the potential for high false-positive rates from screening across all age groups, the task force considers it is not appropriate to recommend population screening in any group aged 65 years or older. Instead, the task force acknowledges the importance of clinical evaluation or case-finding in the context of signs and symptoms to ensure patients are attended to and treated individually.

\section{Cognitive screening instruments}

A systematic review of 14 studies $(n=10185)$ conducted for the US task force,$^{11}$ which was rated as having high methodologic quality (AMSTAR score of 9), reported a sensitivity of $88 \%$ (95\% CI $81 \%$ to $93 \%$ ) and a specificity of $86 \%$ (95\% CI $82 \%$ to $90 \%$ ) for MMSE at cut-off scores of $23 / 24$ or $24 / 25$ to detect dementia. Test performance to detect mild cognitive impairment was based on a smaller body of literature. A recent meta-analysis ${ }^{16}$ also rated as having high methodologic quality (AMSTAR score of 10), reported pooled estimates across 108 studies $(n=$ 36080 ) of $81 \%$ sensitivity (95\% CI $78 \%$ to $84 \%$ ) and $89 \%$ specificity ( $95 \%$ CI $87 \%$ to $91 \%$ ) for MMSE at a cut-off of 23 or 24 to detect dementia; however, the heterogeneity among the studies was large. For the detection of mild cognitive impairment, 21 of the 108 studies $(n=$ 5458) reported MMSE to have $62 \%$ sensitivity (95\% CI $52 \%$ to $71 \%$ ) and $87 \%$ specificity (95\%

Table 2: Summary of recommendations for screening older people ( $\geq 65 \mathrm{yr}$ ) for cognitive impairment from Canada and elsewhere

\begin{tabular}{|ll|}
\hline Organization & \multicolumn{1}{c|}{ Recommendation } \\
\hline $\begin{array}{l}\text { Canadian Task Force on } \\
\text { Preventive Health Care (current) }\end{array}$ & $\begin{array}{l}\text { Do not screen asymptomatic older adults } \\
(\geq 65 \text { yr) for cognitive impairment }\end{array}$ \\
\hline $\begin{array}{l}\text { Canadian Task Force on } \\
\text { Preventive Health Care (2001) })^{15}\end{array}$ & $\begin{array}{l}\text { Insufficient evidence to recommend for or } \\
\text { against screening for cognitive impairment }\end{array}$ \\
\hline $\begin{array}{l}\text { National Institute for Health } \\
\text { and Care Excellence (2011) }\end{array}$ & $\begin{array}{l}\text { Screening for dementia in general } \\
\text { population should not be undertaken }\end{array}$ \\
\hline BC Ministry of Health, 2014 ${ }^{39}$ & Do not screen asymptomatic population \\
\hline $\begin{array}{l}\text { US Preventive Services Task } \\
\text { Force (2014) }\end{array}$ & $\begin{array}{l}\text { Insufficient evidence to assess the balance } \\
\text { of benefits and harms of screening for } \\
\text { cognitive impairment }\end{array}$ \\
\hline
\end{tabular}

CI $80 \%$ to $92 \%) .{ }^{16}$ Across nine studies $(n=$ 1282), MoCA was reported to have a sensitivity of $89 \%$ (95\% CI $80 \%$ to $92 \%$ ) and a specificity of $75 \%$ (95\% CI $62 \%$ to $85 \%$ ) to detect mild cognitive impairment. ${ }^{16}$

Thus, for the MMSE, the likelihood of a false-positive result is $10 \%$ to $14 \%$ when screening for dementia and about $13 \%$ when screening for mild cognitive impairment. For MoCA, screening for mild cognitive impairment could result in a false-positive result in about $25 \%$ of people. Diagnostic accuracy of ADAS-Cog was not reported in the meta-analysis ${ }^{16}$ or in the US task force review, ${ }^{11}$ because the tool is not a brief instrument that could be used in primary care (without substantial training) and could take up to 45 minutes to administer. Because of these reasons, the ADAS-Cog is used mainly in research studies.

The Canadian task force recommends against screening for cognitive impairment in asymptomatic patients; however, practitioners should consider cognitive assessment for patients with signs and symptoms of impairment or when family members or patients express concerns about potential cognitive decline. When deciding whether to screen a patient, clinicians should also consider the potential for false-positive results from screening tools such as MoCA and MMSE. These false-positive results may have implications for harms and costs (e.g., costs related to conducting unnecessary medical care and harms related to treatments).

Finally, although MMSE is a tool with lower false-positive rates than MoCA and is frequently used as a screening test in studies, ${ }^{11,16}$ the tool is available only for professional use with a fee (US\$68 for 50 test forms). The MoCA tool is available to the public without a fee, but it has higher reported false-positive rates than MMSE. ${ }^{12,37}$

\section{Patient values and preferences}

Patient values and preferences were reviewed in the systematic review; ${ }^{10}$ however, no identified Canadian data described the willingness to be screened for or to receive a diagnosis of mild cognitive impairment.

One international study ${ }^{38}$ examined the willingness to be screened among first-degree relatives of people with Alzheimer disease (i.e., children of older people with a diagnosis of probable Alzheimer disease). The study interviewed 93 participants with a mean age of 50.7 years and found that $32 \%$ were willing to be screened within the next year and $42 \%$ during the next five years. Such willingness to be screened was mainly related to obtaining help to prepare for the future.

Participants' responses in terms of factors that 
may influence their willingness to be screened included "help me and my physician plan for future treatments" (57\%); "help me deal with the problem if there was one" (54\%); and "help me plan my life" (52\%). Interestingly, other responses included cost (performing an evaluation is costly, 30\%), time (performing an evaluation is time-consuming, $28 \%$; or it takes time to go see a physician for cognitive impairment screening, 26\%) and other things that are more important for them than screening $(36 \%))^{38}$

Because these participants were relatives of people with a diagnosis of cognitive impairment, it is uncertain whether the findings are generalizable to the broader population of candidates for population screening.

\section{Suggested performance measures}

Given that the task force has recommended against screening, a suggested performance measure for this guideline could be declining use of population screening.

\section{Economic implications}

The task force did not evaluate the economic implications of screening and treatment for cognitive impairment.

\section{Other guidelines}

This new recommendation aligns with other Canadian and international guidelines (Table 2). ${ }^{15,36,39,40}$ The Canadian task force's 2001 guideline stated there was insufficient evidence to recommend for or against screening for cognitive impairment; ${ }^{15}$ the National Institute for Health and Care Excellence $^{36}$ and the BC Ministry of Health ${ }^{39}$ recommend against screening; and the US task force ${ }^{40}$ has concluded that there is insufficient evidence to make a recommendation. It is the practice of the Canadian task force to recommend against the use of a service if there is no evidence that it would be effective.

\section{Gaps in knowledge}

No identified trials directly studied the benefits and harms of screening adults for cognitive impairment. Current evidence evaluating the effect of pharmacologic treatment on mild cognitive impairment was limited to low-quality RCTs of the effect only on cognition. Further research is needed to evaluate other patient-important outcomes, such as function, behaviour and mortality. Research into nonpharmacologic interventions for older people and their families may warrant ongoing investigation. As well, research into how older people value various outcomes and the factors they consider in determining their willingness to be screened will be important for future recommendations on screening and treatment for cognitive impairment. Finally, more research exploring the clinical benefits of screening and treatment in high-risk groups is required.

\section{Conclusion}

The task force recommends not screening community-dwelling asymptomatic older adults ( $\geq 65 \mathrm{yr}$ ) for cognitive impairment. Practitioners should, however, consider cognitive assessment for patients with signs and symptoms of impairment or when family members or patients express concerns about potential cognitive decline.

This recommendation highlights the lack of direct evidence concerning the benefits of screening for cognitive impairment in asymptomatic older adults and the absence of effective treatments for mild cognitive impairment. Furthermore, improved screening tools for mild cognitive impairment are needed. Available screening tools for mild cognitive impairment may incorrectly classify individuals as having the condition (e.g., about 1 in 8 to 10 people with the MMSE and 1 in 4 with MoCA).

\section{References}

1. Glisky EL. Changes in cognitive function in human aging. In: Riddle DR, editor. Brain aging: models, methods, and mechanisms. Boca Raton (FL): CRC Press; 2007. Available: www. ncbi.nlm.nih.gov/books/NBK3885/ (accessed 2015 Nov. 13).

2. What is dementia? Toronto: Alzheimer Society of Canada; 2014. Available: www.alzheimer.ca/en/About-dementia/ Dementias/What-is-dementia (accessed 2013 Oct. 17).

3. Albert MS, DeKosky ST, Dickson D, et al. The diagnosis of mild cognitive impairment due to Alzheimer's disease: recommendations from the National Institute on Aging-Alzheimer's Association workgroups on diagnostic guidelines for Alzheimer's disease. Alzheimers Dement 2011;7:270-9.

4. The health and well-being of Canadian seniors. In: The Chief Public Health Officer's report on the state of public health in Canada 2010. Ottawa: Public Health Agency of Canada; 2010. Available: www.phac-aspc.gc.ca/cphorsphc-respcacsp/2010/ fr-rc/cphorsphc-respcacsp-06-eng.php (accessed 2015 Nov. 13).

5. Manly JJ, Tang M, Schupf N, et al. Frequency and course of mild cognitive impairment in a multiethnic community. Ann Neurol 2008;63:494-506.

6. Ganguli M, Chang CH, Snitz BE, et al. Prevalence of mild cognitive impairment by multiple classifications: the MonongahelaYoughiogheny Healthy Aging Team (MYHAT) project. Am J Geriatr Psychiatry 2010;18:674-83.

7. Katz MJ, Lipton BR, Hall BC, et al. Age-specific and sexspecific prevalence and incidence of mild cognitive impairment, dementia, and Alzheimer dementia in blacks and whites: a report from the Einstein Aging Study. Alzheimer Dis Assoc Disord 2012;26:335-43.

8. Petersen RC, Roberts RO, Knopman DS, et al. Prevalence of mild cognitive impairment is higher in men. The Mayo Clinic Study of Aging. Neurology 2010;75:889-97.

9. Lin JS, O'Connor E, Rossom RC, et al. Screening for cognitive impairment in older adults: an evidence update for the US Preventive Services Task Force. Rockville (MD): Agency for Healthcare Research and Quality; 2013. Report no.: 14-05198-EF-1.

10. Fitzpatrick-Lewis D, Ali MU, Warren R, et al. Screening for mild cognitive impairment: a systematic review. CMAJ Open 2015;3:E419.

11. Lin JS, O'Connor E, Rossom RC, et al. Screening for cognitive impairment in older adults: a systematic review for the US Preventive Services Task Force. Ann Intern Med 2013;159:601-12. 
12. Mini-Mental State Examination. 2nd ed. Lutz (FL): Psychological Assessment Resource (PAR); 2012.

13. Frequently asked questions. Montréal: Montreal Cognitive Assessment; 2015. Available: www.mocatest.org/faq/ (accessed 2015 May 28).

14. Tricco AC, Soobiah C, Berliner S, et al. Efficacy and safety of cognitive enhancers for patients with mild cognitive impairment: a systematic review and meta-analysis. CMAJ 2013;185:1393-401.

15. Patterson CJ, Gass DA. Screening for cognitive impairment and dementia in the elderly. Can J Neurol Sci 2001;28(Suppl 1): S42-51.

16. Tsoi KK, Chan JY, Hirai HW, et al. Cognitive tests to detect dementia: a systematic review and meta-analysis. JAMA Intern Med 2015;175:1450-8.

17. Connor Gorber S, Singh H, Pottie K, et al. Process for guideline development by the reconstituted Canadian Task Force on Preventive Health Care. CMAJ 2012;184:1575-81.

18. Schünemann H, Brożek J, Guyatt $\mathrm{G}$, et al., editors. GRADE handbook for grading the quality of evidence and strength of recommendations. Updated October 2013. The GRADE Working Group; 2013. Available: www.guidelinedevelopment.org/ handbook.

19. Doody RS, Ferris SH, Salloway S, et al. Donepezil treatment of patients with MCI: a 48-week randomized, placebo-controlled trial. Neurology 2009;72:1555-61.

20. Petersen RC, Thomas RG, Grundman M, et al. Vitamin E and donepezil for the treatment of mild cognitive impairment. N Engl J Med 2005;352:2379-88

21. Feldman $\mathrm{HH}$, Ferris S, Winblad B, et al. Effect of rivastigmine on delay to diagnosis of Alzheimer's disease from mild cognitive impairment: the InDDEx study. Lancet Neurol 2007;6:501-12.

22. Winblad B, Gauthier S, Scinto L, et al. Safety and efficacy of galantamine in subjects with mild cognitive impairment. $\mathrm{Neu}$ rology 2008;70:2024-35

23. de Jager CA, Oulhaj A, Jacoby R, et al. Cognitive and clinical outcomes of homocysteine-lowering B-vitamin treatment in mild cognitive impairment: a randomized controlled trial. Int $J$ Geriatr Psychiatry 2012;27:592-600.

24. Lee LK, Shahar S, Chin A, et al. Docosahexaenoic acidconcentrated fish oil supplementation in subjects with mild cognitive impairment (MCI): a 12-month randomised, double-blind, placebo-controlled trial. Psychopharmacology (Berl) 2013;225: 605-12.

25. Naeini AM, Elmadfa I, Djazayery A, et al. The effect of antioxidant vitamins $\mathrm{E}$ and $\mathrm{C}$ on cognitive performance of the elderly with mild cognitive impairment in Isfahan, Iran: a double-blind, randomized, placebo-controlled trial. Eur J Nutr 2014;53:1255-62.

26. Rojas GJ, Villar V, Iturry M, et al. Efficacy of a cognitive intervention program in patients with mild cognitive impairment. Int Psychogeriatr 2013;25:825-31.

27. Suzuki T, Shimada H, Makizako H, et al. Effects of multicomponent exercise on cognitive function in older adults with amnestic mild cognitive impairment: a randomized controlled trial. BMC Neurol 2012;12:128.

28. Suzuki T, Shimada H, Makizako $\mathrm{H}$, et al. A randomized controlled trial of multicomponent exercise in older adults with mild cognitive impairment. PLOS ONE 2013;8:e61483.

29. Tsolaki M, Kounti F, Agogiatou C, et al. Effectiveness of nonpharmacological approaches in patients with mild cognitive impairment. Neurodegener Dis 2011;8:138-45.

30. Wei XH, Ji L. Effect of handball training on cognitive ability in elderly with mild cognitive impairment. Neurosci Lett 2014; 566:98-101.

31. Huntley JD, Gould RL, Liu K, et al. Do cognitive interventions improve general cognition in dementia? A meta-analysis and meta-regression. BMJ Open 2015;5:e05247.

32. Rondanelli M, Opizzi A, Faliva M, et al. Effects of a diet integration with an oily emulsion of DHA-phospholipids containing melatonin and tryptophan in elderly patients suffering from mild cognitive impairment. Nutr Neurosci 2012;15:46-54.

33. Yakoot M, Salem A, Helmy S. Effect of $\mathrm{Memo}^{\oplus}$, a natural formula combination, on Mini-Mental State Examination scores in patients with mild cognitive impairment. Clin Interv Aging 2013;8:975-81.

34. Tsai PF, Chang JY, Beck C, et al. A pilot cluster randomized trial of a 20 -week Tai Chi program in elders with cognitive impairment and osteoarthritic knee: effects on pain and other health outcomes. J Pain Symptom Manage 2013;45:660-9.

35. van Uffelen JGZ, Chinapaw MJM, van Mechelen W, et al. Walking or vitamin B for cognition in older adults with mild cognitive impairment? A randomised controlled trial. $\mathrm{Br} J$ Sports Med 2008;42:344-51.

36. National Collaborating Centre for Mental Health. Dementia: supporting people with dementia and their carers in health and social care. London (UK): National Institute for Health and Care Excellence; 2011:42.

37. MoCA test full. Montréal: Montreal Cognitive Assessment; 2015. Available: www.mocatest.org/paper-tests/moca-test-full/ (accessed 2015 July 21)

38. Werner P. Beliefs about memory problems and help seeking in elderly persons. Clin Gerontol 2004;27:19-30.

39. Cognitive impairment: recognition, diagnosis and management in primary care. Vancouver: BC Ministry of Health, Guidelines and Protocols Advisory Committee; 2014; Available: http://www2 .gov.bc.ca/gov/content/health/practitioner-professional-resources/ bc-guidelines/cognitive-impairment (accessed 2015 Apr. 28)

40. Moyer VA; US Preventive Services Task Force. Screening for cognitive impairment in older adults: US Preventive Services Task Force recommendation statement. Ann Intern Med 2014; 160:791-7.

Guideline writing group: Kevin Pottie, Rana Rahal, Alejandra Jaramillo, Richard Birtwhistle, Brett D. Thombs, Harminder Singh, Sarah Connor Gorber, Lesley Dunfield, Amanda Shane, Maria Bacchus, Neil Bell and Marcello Tonelli.

Affiliations: Departments of Family Medicine, and of Epidemiology and Community Medicine, University of Ottawa, and Bruyère Research Institute (Pottie), Ottawa, Ont; Public Health Agency of Canada (Rahal, Jaramillo, Connor Gorber, Dunfield, Shane), Ottawa, Ont.; Departments of Family Medicine and of Community Health and Epidemiology, and Centre for Studies in Primary Care (Birtwhistle), Queen's University, Kingston, Ont.; Department of Medicine, Lady Davis Institute, Jewish General Hospital and Faculty of Medicine, McGill University (Thombs), Montréal, Que.; Department of Internal Medicine and Community Health Sciences (Singh), University of Manitoba, Winnipeg, Man.; Department of Family Medicine (Bell), University of Alberta, Edmonton, Alta.; Department of Medicine (Bacchus, Tonelli), University of Calgary, Calgary, Alta.

Contributors: All of the authors contributed substantially to the interpretation of the findings. Kevin Pottie, Sarah Connor Gorber, Rana Rahal, Alejandra Jaramillo and Marcello Tonelli drafted the article with assistance from the rest of the guideline writing group. All of the authors approved the final version to be published and agreed to act as guarantors of the work.

Funding: Funding for the Canadian Task Force on Preventive Health Care is provided by the Public Health Agency of Canada and the Canadian Institutes of Health Research. The views of the funding bodies have not influenced the content of the guideline; competing interests have been recorded and addressed. The views expressed in this article are those of the authors and do not necessarily represent those of the Public Health Agency of Canada.

Acknowledgements: The authors acknowledge members of the McMaster Evidence Review and Synthesis Centre (Donna Fitzpatrick-Lewis, Muhammad Usman Ali, Rachel Warren, Diana Sherifali, Parminder Raina, Maureen Rice, Leslea Peirson, Meghan Kenny and Sharon Peck-Reid) for their expert search, selection and synthesis of evidence on screening and full systematic review on treatment. 